\title{
An Empirical Model of Behavioral Heterogeneity
}

\author{
SUBAL C. KUMBHAKAR \\ SUNY-Binghampton* \\ MIKE G. TSIONAS \\ Lancaster University and Athens University
}

\begin{abstract}
In this paper we propose a new latent class/mixture model (LCM) to determine whether firms behave like profit maximizers or just cost minimizers when there is no additional sample separation information. Since some firms might be maximizing profit while others might minimize cost, the LCM with behavioral heterogeneity can be quite useful. Estimation of the LCM amounts to mixing a cost minimization and a profit maximization model. Using the U.S. airlines data we find that after deregulation about $15 \%$ of the airlines are found to be consistent with profit maximizing behavior.
\end{abstract}

JEL Classification.: C51, L93, L2

Keywords: Cost minimization, profit maximization, latent class, US airlines.

\section{Introduction}

Cost minimization and profit maximization behavioral assumptions are most widely used in microeconomic theory to analyze firm behavior. However, in practice researchers do not know whether every firm in the sample maximizes profit or minimizes cost. In this paper we address this problem via a latent class modeling approach in which some producers minimize cost while others also maximize profit, and probabilities of being in these groups are made functions of covariates. This approach does not require researchers to know which firms maximize profit. The model helps us to determine not only which firms behave like profit maximizers but also what differentiates them from firms that failed to maximize profit. The new technique is illustrated using data on US airlines. The empirical findings suggest that very few units maximize profit.

\footnotetext{
Kumbhakar: Department of Economics, State University of New York - Binghamton, kkar@,binghamton.edu. Tsionas: Department of Economics, Lancaster University Management School and Department of Economics, Athens University of Economics and Business, m.tsionas@1ancaster.ac.uk

(C) 2016 Subal C. Kumbhakar and Mike G. Tsionas. Licensed under the Creative Commons Attribution - Noncommercial 3.0 Licence (http://creativecommons.org/licenses/by$\mathrm{nc} / 3.0 /$. Available at http: //rofea.org.
} 


\section{The classical optimization problems}

We start with the standard cost function approach ${ }^{1}$ that is based on the assumption that producers minimize cost, given output and input prices. In this approach one specifies a cost function and derives the cost share equations (input demand functions) using Shephard's lemma. Usually a translog cost function is chosen to represent the underlying production technology. The corresponding cost system (Christensen and Greene (1976)) is then written as

$$
\begin{aligned}
& \ln C_{i}=\ln C\left(\ln p_{i}, \ln y_{i}\right)+v_{1 i} \\
& S_{1 i}=S_{1}\left(\ln p_{i}, \ln y_{i}\right)+v_{2 i} \\
& \vdots \\
& S_{M-1, i}=S_{M-1}\left(\ln p_{i}, \ln y_{i}\right)+v_{M i}
\end{aligned}
$$

where $C_{i}$ is total cost, $S_{1 i}, \ldots, S_{M-1, i}$ denote the $M-1$ cost shares $^{2}, p_{i}$ is the $M \times 1$ vector of input prices, $y_{i}$ is the $Q \times 1$ vector of outputs, and $v_{i}=\left[v_{1 i}, \ldots, v_{M i}\right]^{\prime}$ represents the error vector. The subscript $i(i=1, \ldots, N)$ indicates producers/firms. If $v_{i} \sim I N_{M}\left(0_{M}, \Omega\right)$ where $\Omega$ is the $M \times M$ covariance matrix, the joint density of the cost system in (1) can then be written as

$$
f_{Z_{i}}\left(Z_{i}\right)=(2 \pi)^{-M / 2}\left(\operatorname{det} \Omega^{-1}\right)^{1 / 2} \exp \left(-\frac{1}{2} v_{i}^{\prime}\left(Z_{i} ; \theta\right) \Omega^{-1} v_{i}\left(Z_{i} ; \theta\right)\right)
$$

where

$$
Z_{i}=\left[\ln C_{i}, S_{1 i}, \ldots, S_{M-1, i}\right]^{\prime}
$$

and

$$
v_{i}\left(Z_{i} ; \theta\right)=\left[\ln C_{i}-\ln C\left(\ln p_{i}, \ln y_{i}\right), S_{1 i}-S_{1}\left(\ln p_{i}, \ln y_{i}\right), \ldots, S_{M-1, i}-S_{M-1}\left(\ln p_{i}, \ln y_{i}\right)\right]^{\prime}
$$

\footnotetext{
${ }^{1}$ Beard, Caudill and Gropper (1997) considered mixing cost functions to study differences in technology across regimes. They assumed cost minimizing behavior for all observations but allowed the technology to differ across regimes. See also Orea and Kumbhakar (2004) for a stochastic cost frontier application.

${ }^{2}$ One cost share is dropped to avoid the singularity problem.
} 


\section{KUMBHAKAR, TSIONAS Empirical Model of Behavioral Heterogeneity}

Under profit maximization, we have $Q$ additional choice variables - the optimum values of which are to be obtained from the $Q$ additional conditions, viz., $q_{j}=\partial C / \partial y_{j} \quad j=1, \ldots, Q$ ), where $q_{j}$ is the price of output $y_{j}$. These first-order conditions for profit maximization state that output allocation is optimal when output price equals marginal cost. These equations can be rewritten as

$$
\ln y_{j i}=\ln C_{i}-\ln q_{j i}+\ln \left(e c y_{j i}\left(\ln p_{i}, \ln y_{i}\right)\right)+v_{M+j, i}, j=1, \ldots, Q, i=1, \ldots, N
$$

where $e c y_{j i}\left(\ln p_{i}, \ln y_{i}\right)=\frac{\partial \ln C\left(\ln p_{i}, \ln y_{i}\right)}{\partial \ln y_{j i}}$ is the output elasticity. Under the behavioral assumption of profit maximization, these additional conditions in (3) are to be appended to the cost system in (1) so that we have a complete system of $M+Q$ equations for $M+Q$ endogenous (choice) variables ( $M$ inputs $^{3}$ and $Q$ outputs). Another difference with the cost system in (1) is that the present system for the profit maximizing model consisting of (1) and (3) can no longer be estimated using the SUR technique. ${ }^{4}$ This is because the endogenous variables (especially outputs) appear on both sides of the equations in (1) and (3). The endogenous variables of the profit system in vector form are:

$$
\Xi_{i}=\left[\ln C_{i}, S_{i}^{\prime}, \ln y_{i}^{\prime}\right]^{\prime}
$$

where

$$
S_{i}=\left[S_{1 i}, \ldots, S_{M-1, i}\right]^{\prime} \text {, and } \ln y_{i}=\left[\ln y_{1 i}, \ldots, \ln y_{Q i}\right]^{\prime}
$$

Let:

$$
v_{i}=\left[v_{1 i}, \ldots, v_{M+Q, i}\right]^{\prime} \sim I N_{M+Q}\left(0_{M+Q}, \Sigma\right)
$$

where $\Sigma$ is an $(M+Q) \times(M+Q)$ covariance matrix. We have a nonlinear simultaneous equation model that can be written in the form $f\left(\Xi_{i}, \Psi_{i} ; \theta\right)=v_{i}, i=1, \ldots, N$, where $\Psi_{i}$ represents the vector of predetermined variables (prices, and possibly other quasi-fixed factors or shift variables) and $\theta \in \Theta \subseteq R^{k}$ is the parameter vector. The above notation is appropriate for an implicit nonlinear system.

\footnotetext{
${ }^{3}$ The cost system in (1) treats the cost and (J-1) cost shares as endogenous variables. This is equivalent to treating the inputs as endogenous.

${ }^{4}$ Equation (3) is often written as $R_{j i}=q_{j i} y_{j i} / C_{i}=e c y_{j i}+v_{M+j, i}$ and then jointly estimated together with equation in (1) as a SUR system. This is wrong because the SUR equations do not recognize that $y$, appearing on the right hand side in these equations, is endogenous.
} 
The joint density function of endogenous variables is

$$
f_{\Xi_{i}}\left(\Xi_{i} ; \theta\right)=f_{v_{i}}\left(v_{i}\left(\Xi_{i}, \Psi_{i} ; \theta\right)\right) \cdot\left|J\left(\Xi_{i}, \Psi_{i} ; \theta\right)\right|
$$

where $\left|J\left(\Xi_{i}, \Psi_{i} ; \theta\right)\right|=\left|\left(\frac{\left.\partial v_{i}\left(\Xi_{i}, \Psi_{i} ; \theta\right)\right)}{\partial \Xi_{i}}\right)\right|$ is the absolute value of the determinant of the Jacobian of the transformation,

$f_{v_{i}}\left(v_{i}\left(\Xi_{i}\right)\right)=(2 \pi)^{-(M+Q) / 2}\left(\operatorname{det} \Sigma^{-1}\right)^{1 / 2} \exp \left(-\frac{1}{2} v_{i}^{\prime}\left(\Xi_{i}, \Psi_{i} ; \theta\right) \Sigma^{-1} v_{i}\left(\Xi_{i}, \Psi_{i} ; \theta\right)\right), i=1, \ldots, N$

and $v_{i}\left(\Xi_{i}, \Psi_{i} ; \theta\right) \equiv f\left(\Xi_{i}, \Psi_{i} ; \theta\right)$. The Jacobian is:

$$
\left|\operatorname{det}\left(\frac{\left.\partial v_{i}\left(\Xi_{i}, \Psi_{i} ; \theta\right)\right)}{\partial \Xi_{i}}\right)\right|=\left|1-e c y_{i}^{-1} \frac{\partial e c y_{i}}{\partial \Xi_{i}}-e c y_{i}\right|=\left|1-e c y_{i}^{-1} a_{y y}-e c y_{i}\right|, i=1, . ., N
$$

where $e c y_{i}=\operatorname{diag}\left[e c y_{1 i}, \ldots, e c y_{Q i}\right]^{\prime}, e c y_{i}^{-1}=\operatorname{diag}\left[1 / e c y_{1 i}, \ldots, 1 / e c y_{Q i}\right]^{\prime}$, and $1_{Q}$ is the $Q \times 1$ unit vector.

\section{Behavioral latent class model}

Kulatilaka (1985) developed tests that can be used to test the significance of departures from the first order condition that price equals marginal cost at each data point. The test developed by Schankerman and Nadiri (1986). This test determines whether producers (as a whole) maximize profit or simply minimize cost.

The alternative approach explored here is to assume that every producer is potentially a profit maximizer (with some probability). The probability of being a profit maximizer is specified by a logistic function that depends on some exogenous variables. This gives us a finite mixture/latent class model (McLachlan and Peel (2000)), where the density of endogenous variables is given by

$$
f\left(\Xi_{i} ; \theta\right)=\pi_{i} f_{\Xi_{i}}\left(\Xi_{i} ; \theta\right)+\left(1-\pi_{i}\right) p_{Z_{i}}\left(Z_{i} ; \theta\right), i=1, \ldots, N
$$

where $\pi_{i}$ is the probability that the $i$ th firm behaves as if it were profit maximizing. Recall that $\Xi_{i}$ includes $\log$ cost, shares, and $\log$ outputs, and $Z_{i}$ includes $\log$ cost and shares, exclusively. So $Z_{i}$ is a subset of $\Xi_{i}$. However, a formal comparison of $f_{\Xi_{i}}\left(\Xi_{i} ; \theta\right)$ and $p_{Z_{i}}\left(Z_{i} ; \theta\right)$ is not possible since they refer to different endogenous variables. It is, however, 
KUMBHAKAR, TSIONAS Empirical Model of Behavioral Heterogeneity

quite reasonable to compare the cost minimization density $p_{Z_{i}}\left(Z_{i} ; \theta\right)$ with the implied cost minimization density of the profit maximization system, that is, $p_{Z_{i}}^{C P M}\left(Z_{i} \mid \ln y_{i}, \theta\right)$. The density can be written as

$$
p_{Z_{i}}^{C P M}\left(Z_{i} \mid \ln y_{i}, \theta\right)=\frac{f_{\Xi_{i}}\left(\Xi_{i} ; \theta\right)}{f_{\ln y_{i}}\left(\ln y_{i} ; \theta\right)}
$$

Although the numerator is available from the profit maximization problem, the denominator is

$$
f_{\ln y_{i}}\left(\ln y_{i} ; \theta\right)=\int_{\square M} f_{\Xi_{i}}\left(\Xi_{i} ; \theta\right) d Z_{i}
$$

Recall that $\Xi_{i}=\left[\begin{array}{c}Z_{i} \\ \ln y_{i}\end{array}\right]$ and, therefore, we have to integrate log cost and shares out of the profit maximization system (PMM).

To compute the implied cost minimization density, we have to apply numerical integration to (6) with respect to $Z_{i}$ s. The integral cannot be computed by "completing the square" as usual, because of the nonlinearity of $v_{i}\left(\Xi_{i}, \Psi_{i} ; \theta\right)$ with respect to $Z_{i}$ s. The nonlinearity is caused by the term $\ln e c y_{i}$ in (3).

Given a set of predetermined variables, $W_{i}$, we parameterize the log-odds ratio in favor of profit maximization as follows:

$$
\ln \left(\frac{\pi_{i}}{1-\pi_{i}}\right)=W_{i}^{\prime} \delta, i=1, \ldots, N
$$

where $\delta \in \Delta \subseteq R^{h}$ is a vector of parameters. Using (6) we can formulate the log-likelihood function

$$
\ln L\left(\theta, \Sigma^{-1}, \Omega^{-1}, \delta ; \Xi, \Psi, W\right)=\sum_{i=1}^{N} \ln \left[\pi_{i} f_{\Xi_{i}}\left(\Xi_{i} ; \theta\right)+\left(1-\pi_{i}\right) p_{Z_{i}}^{C P M}\left(Z_{i} ; \theta\right)\right]
$$

We can maximize this function to obtain FIML estimates of all parameters. Straightforward application of Bayes' theorem yields an estimate of the posterior probability that the $i$ th firm maximizes profit: 


$$
\tilde{Q}_{i}=\frac{\pi_{i}(\tilde{\delta}) f_{\Xi_{i}}\left(\Xi_{i} ; \tilde{\theta}\right)}{\pi_{i}(\tilde{\delta}) f_{\Xi_{i}}\left(\Xi_{i} ; \tilde{\theta}\right)+\left(1-\pi_{i}(\tilde{\delta})\right) p_{Z_{i}}^{C P M}\left(Z_{i} ; \tilde{\theta}\right)}, i=1, \ldots, N,
$$

where the FIML estimates were substituted for $\theta$ and $\delta$, and

$$
\pi_{i}(\widetilde{\delta}) \equiv \frac{\exp \left(W_{i} \widetilde{\delta}\right)}{1+\exp \left(W_{i} \widetilde{\delta}\right)}, i=1, \ldots, N
$$

These posterior probabilities are firm-specific even when $\pi_{i}$ is a parameter. Ideally, we would like to have $\tilde{Q}_{i}$ equal to either zero or one (or nearly so) so that the choice in favor or against profit maximization is more or less clear. Empirically, we cannot always expect that and therefore one has to use a cut-off probability (e.g., $\tilde{Q}_{i}>$.5) to determine whether a firm falls in the profit maximizer group or not.

\section{Empirical application}

To illustrate the technique proposed in the preceding sections, we use an unbalanced panel data set (for details see Baltagi et al. (1998)) consisting of annual observations on the domestic operations of 23 US airlines over the period 1971-1986. This gives us a total of 268 observations. Variable inputs are: labor $(L)$, materials $(M)$ and fuel $(F)$. Capital $(K)$ it treated as a quasi-fixed factor. To control for firm-heterogeneity, we also include 22 airline dummies in the cost function. In the log-odds ratio, we used a constant term, a deregulation dummy and 22 airline dummies. From the estimated coefficients in the log-odds equation, we found that (i) all the coefficients are statistically significant, (ii) deregulation has a statistically significant and positive impact upon the odds in favor of profit maximization, and (iii) airlines seem to differ substantially in their individual log-odds in favor of profit maximization.

Mean values of scale economies are found to be $0.56,1.037$ and 0.552 for the CMM, the PMM and the LCM, respectively, with standard deviations 0.06, 0.301, and 0.130. Since the Schankerman-Nadiri test rejects the overall profit maximizing behavior, results from the PMM might be misleading. To highlight these differences and examine their behavior before and after deregulation in 1977, we report kernel density plots of output elasticities for these three models (before and after deregulation) in Figures 1(a) and 1(b). It can be seen from these figures that distributions of output elasticities are significantly different from one another. The distribution from the cost system is extremely tight, while for the profit system it is just the opposite. The results from the mixture model are in between. Furthermore, the qualitative nature of these distributions has not been changed after deregulation. 


\section{KUMBHAKAR, TSIONAS Empirical Model of Behavioral Heterogeneity}

The mean technical change from the CMM, PMM and LCM are found to be -0.029 , 0.036 , and -0.028 , respectively, with standard deviations $0.005,0.003$, and 0.001 . Thus, on average, technical progress took place at the rate of $2.8 \%$ to $3.6 \%$ per annum. To focus more on these differences, kernel density estimates of airline-specific measures of technical change are reported in Figures (1c) (before regulation) and (1d) (after deregulation). Although deregulation is not found to have a large impact but differences across models seem to be substantial. The spread of technical change distribution is found to be the highest for the CMM (opposite of output elasticity distribution) followed by the PMM. The distribution is very tight for the LCM. These results are true for both pre- and post-deregulation regimes.

We prefer the LCM because it allows us to estimate the same technology irrespective of whether an airline maximizes profit or not. In other words, the LCM can impose the constraint of common technology irrespective of behavioral assumptions. Although the mean technical change from the PMM is not very different from the other two models, distributions of technical change obtained from the PMM, CMM and the LCM are quite different.

From estimated posterior probabilities (see also Figure 2) only the following observations seem to favor profit maximization behavioral assumption: BR (1981), CN (1984-1986), ML (1982-1986), NW (1972-1986), PA (1986), PO (1986), and WN. Only NW and WN consistently behaved like profit maximizers. The posterior probability in favor of profit maximizing behavior increases somewhat (from about $8 \%$ to $15 \%$ ) after the deregulation but the bulk of airlines remain cost minimizers. Thus, we do not have clear evidence that deregulation changed economic behavior of the airlines, viz., from cost minimization to profit maximization.

\section{Conclusions}

In this paper we proposed a new latent class/mixture model (LCM) to determine whether firms behave like profit maximizers or just cost minimizers without sample separation information. The Schankerman and Nadiri (1989) test gives an overall conclusion of either in favor or against profit maximization for all firms in the sample. In practice some firms might be maximizing profit while others might minimize cost. In such a situation the LCM with behavioral heterogeneity can be quite useful. Estimation of the LCM amounts to mixing a cost system with a profit system in such a way that the technology is the same for all firms irrespective of their behavioral assumptions. Estimates of the log-odds ratio are used to predict the posterior probability of U.S. airlines maximizing profit or minimizing cost. We find that after deregulation about $15 \%$ of the airlines are found to be consistent with profit maximizing behavior. In other words, we do not find evidence that all airlines were maximizing profit, especially after deregulation in 1978. 


\section{References}

Baltagi, B.H., J.M. Griffin, and S.R. Vadali (1998), Excess capacity: A permanent characteristic of US airlines? Journal of Applied Econometrics 13, 645-657.

Beard, T., S. Caudill, and D. Grooper (1997), The Diffusion of Production Processes in the U.S. Banking Industry: A Finite Mixture Approach, Journal of Banking and Finance 21, 721-740.

Christensen, L. R., and W. H. Greene (1976), Economies of Scale in U. S. Electric Power Generation, Journal of Political Economy 84:4, 655-76.

Kulatilaka, N. (1985), Tests on the validity of static equilibrium models, Journal of Econometrics 28, 253-268.

McLachlan, G.J. and D. Peel (2000), Finite Mixture Models, John Wiley \& Sons, New York..

Orea, L. and S.C. Kumbhakar (2004), Efficiency Measurement using a Latent Class Stochastic Frontier Model, Empirical Economics, 29, pp. 169-183.

Schankerman, M., and M.I. Nadiri (1986), A test of static equilibrium models and rates of return to quasi-fixed factors, with an application to the Bell system, Journal of Econometrics 33, 97-118. 
Figure 1 : Cost elasticity and technical change

10. Qutput east elastinity befare deregulatian

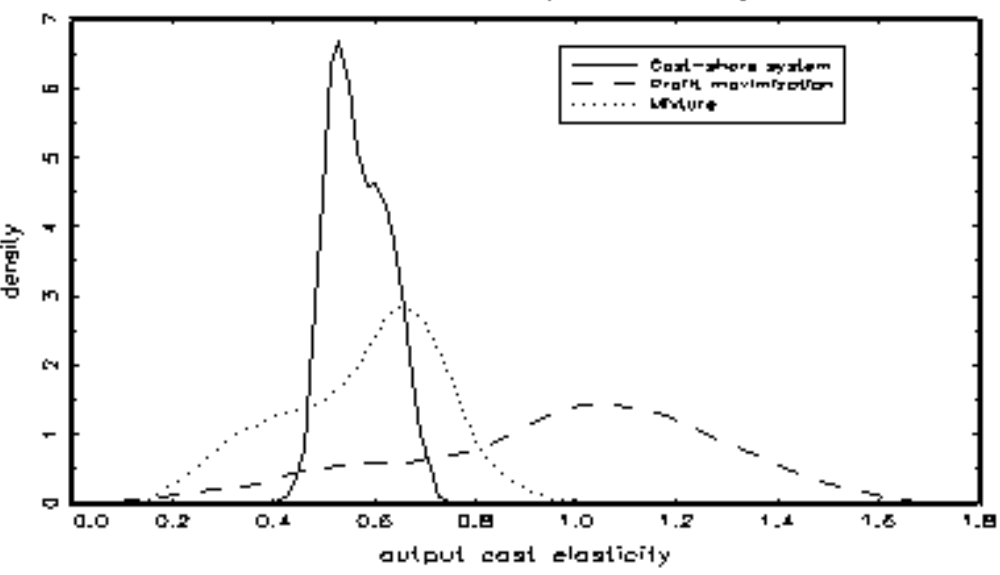

1.. Technizal change befare deregulatian

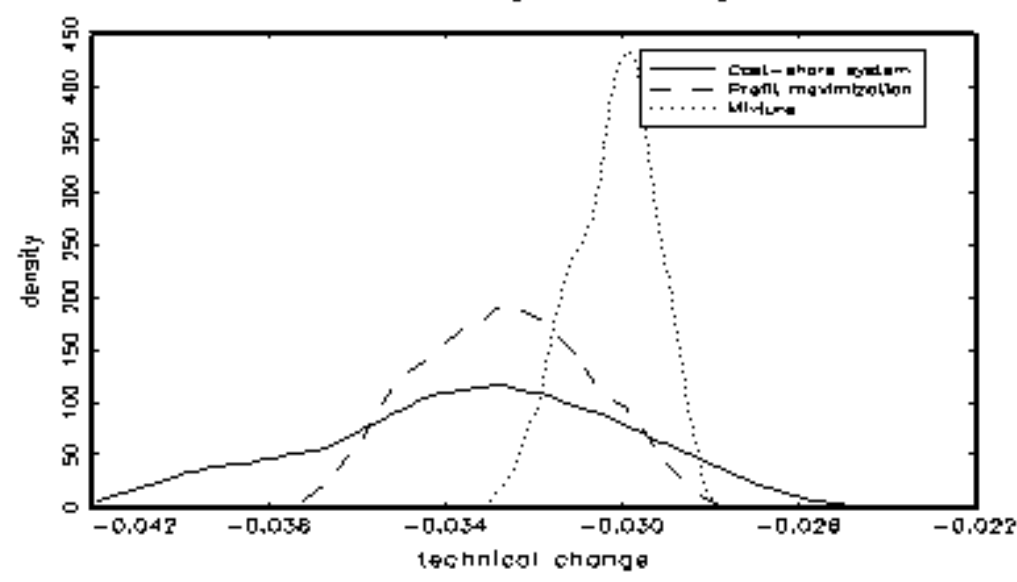

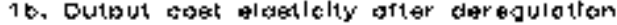

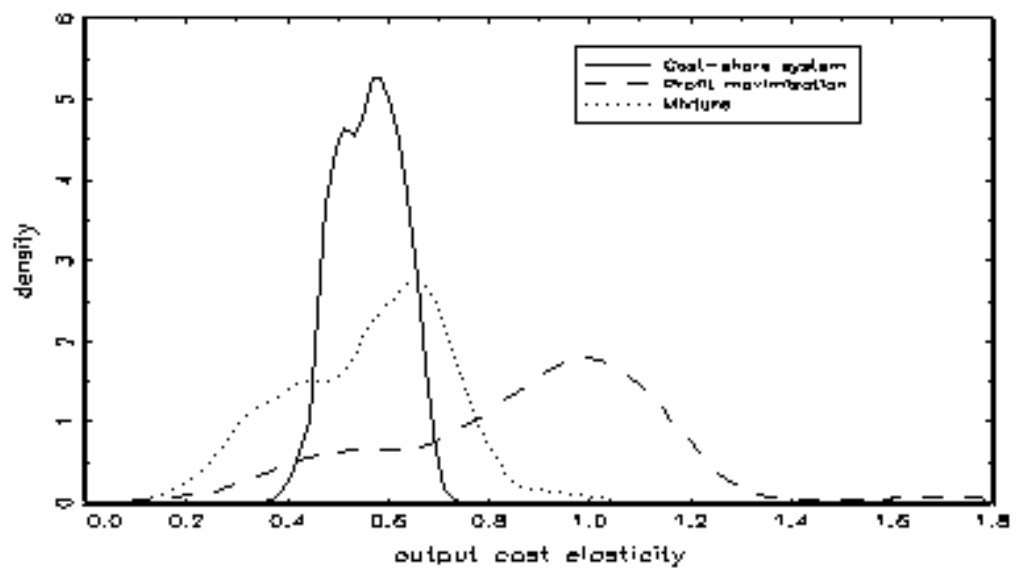

1d. Tectinleal eharige after deregulatlan

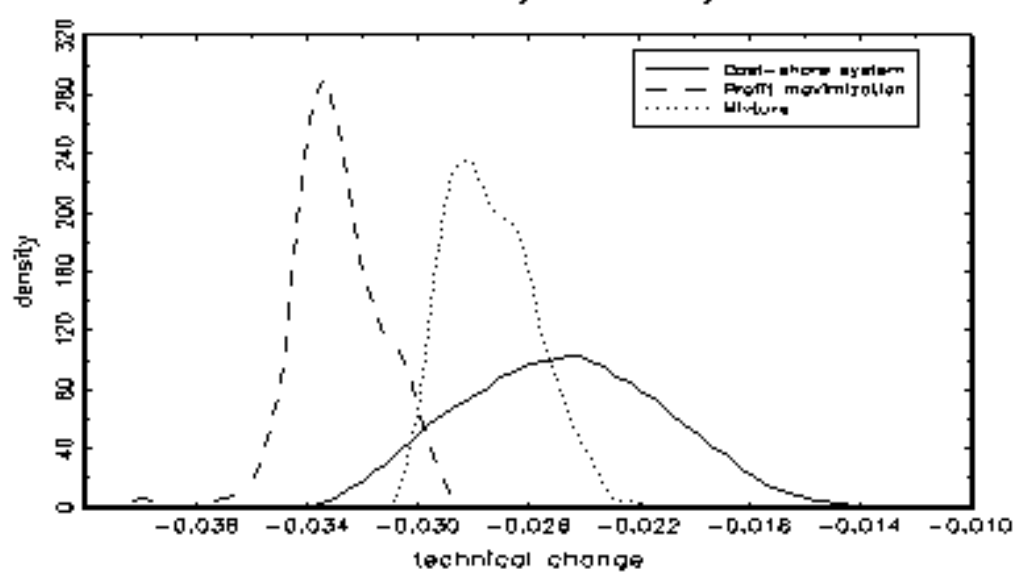


Review of Economic Analysis 8 (2016) 125-134

Figure 2: Probability of profit maximization

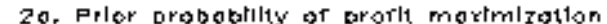

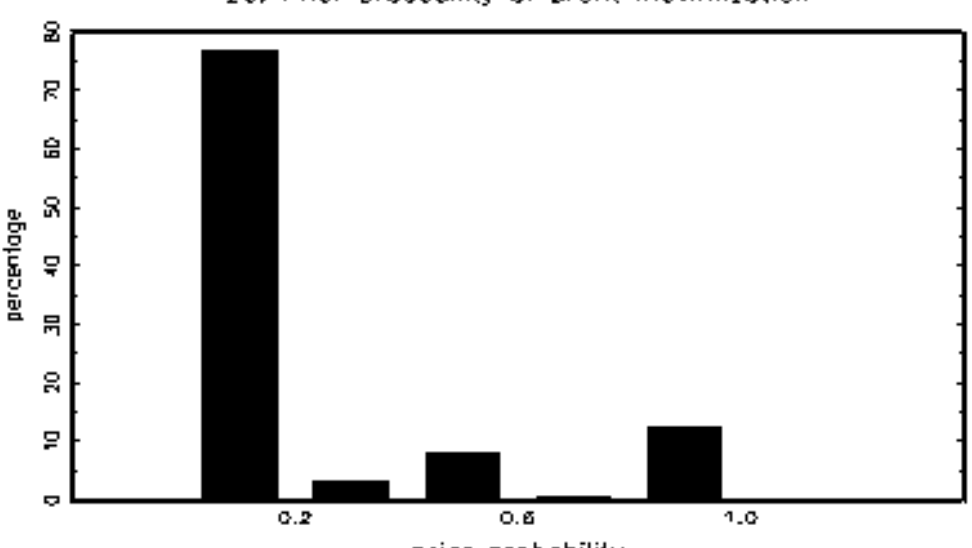

priar pratability

20. Fogtertar aroboblity berore dergulotan

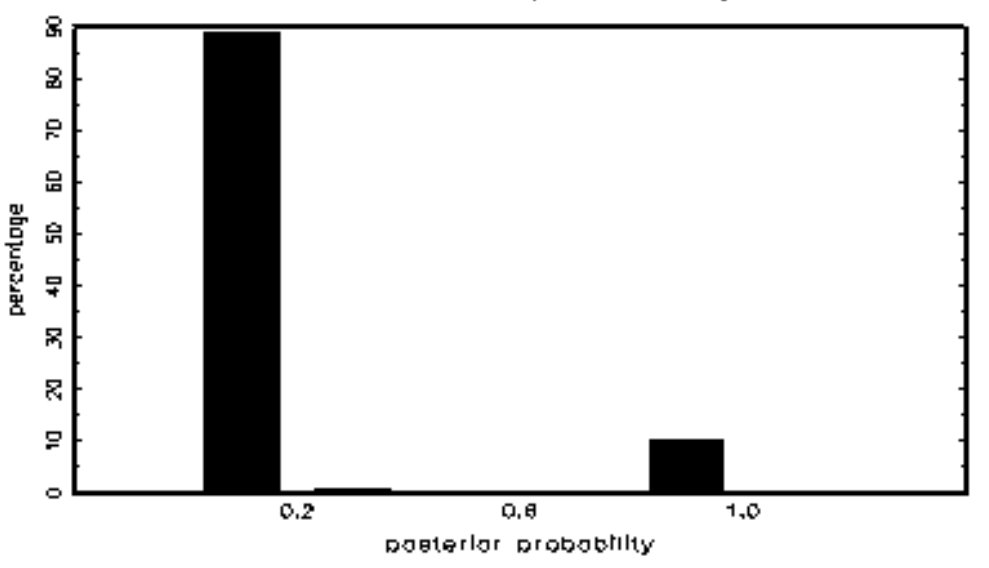

2t. Owerall pagleriar probatility of prant maximizalian

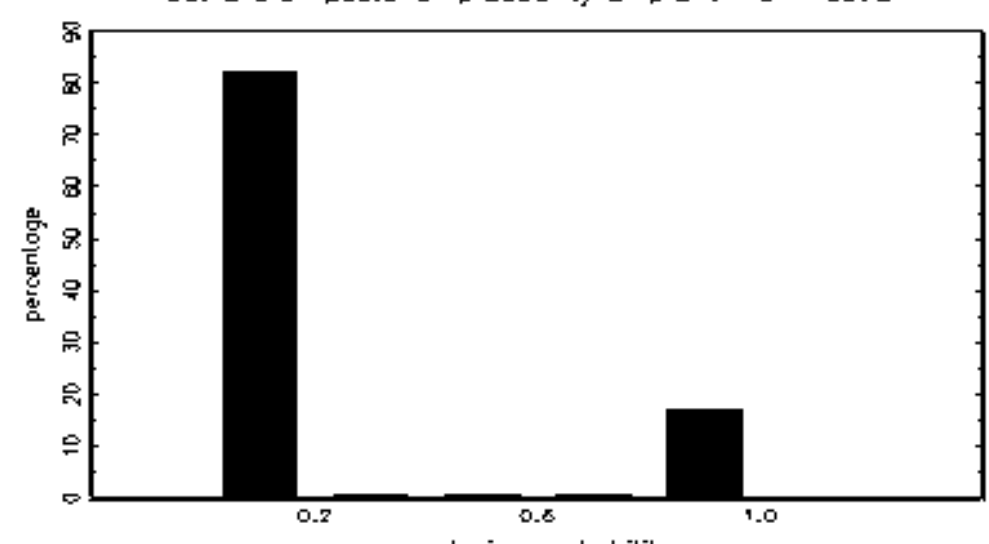

Fosterior prabability

2d. Pasterigr prabability orter deregulatign

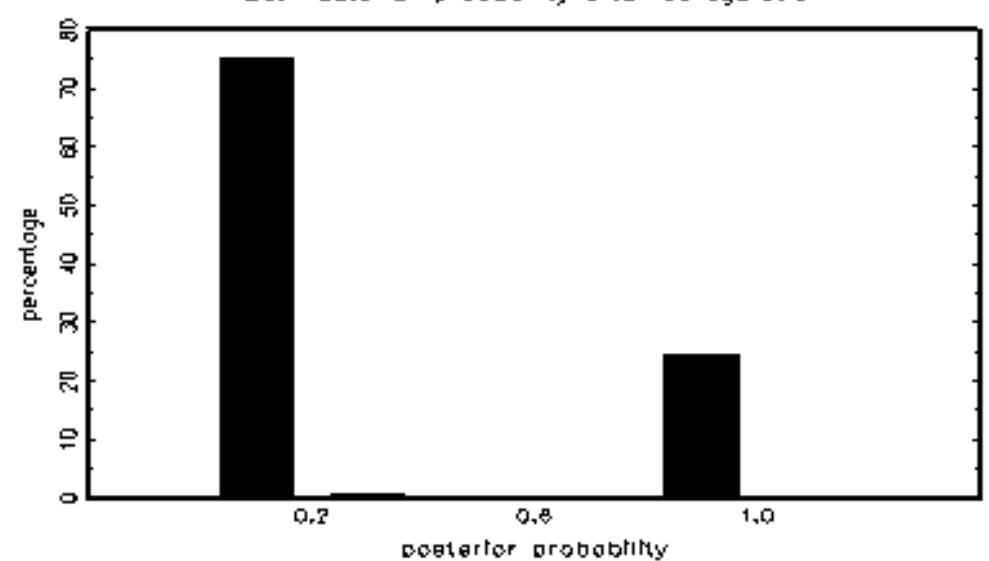

